\title{
Self-Report Naps in Irregular Work Schedules
}

\author{
June J. PILCHER ${ }^{1 *}$, Stephen M. POPKIN ${ }^{2}$, Karen ADKINS ${ }^{1}$ and Lindsay ROETHER ${ }^{1}$ \\ ${ }^{1}$ Department of Psychology, Clemson University, 418 Brackett Hall, Clemson University, Clemson, SC 29634- \\ 1355, USA \\ ${ }^{2}$ Volpe National Transportation Systems Center, Cambridge, MA 02142, USA
}

Received September 22, 2004 and accepted November 26, 2004

\begin{abstract}
Few studies have examined napping in irregular work schedules such as those experienced by freight locomotive engineers. The current study examined the effect of napping in irregular work schedules on sleep length and three subjective measures: ability to go to sleep, ability to stay asleep, and a feeling of being well-rested upon awakening. One hundred and seventy-nine freight engineers completed a 14-d activity log, providing information on sleep times and subjective evaluations of sleep. The results indicated that days with naps resulted in significantly more total sleep but less sleep in the main sleep period of the day. The days with naps also resulted in somewhat more difficulty with going to sleep, staying asleep and with feeling well-rested upon awakening. It is important to note, however, that when examining the days with naps, nap length was not significantly correlated with main sleep time, ability to go to sleep, ability to stay asleep, or feeling well-rested upon awakening. These results suggest that napping may be useful when working irregular work schedules if the engineer is willing to accept a slight decrease in ability to go to sleep, stay asleep, and feeling rested.
\end{abstract}

Key words: Irregular work schedules, Shift work, Napping, Sleep quantity, Sleep quality, Train drivers', Circadian rhythms

\section{Introduction}

There has been increased interest in recent years in the potential use of napping to maximize alertness when awake. For example, some studies have examined the use of naps as a potential countermeasure for sleepiness associated with shift work ${ }^{1-3)}$ or with prolonged wakefulness ${ }^{4-6}$. Previous studies have also shown that napping can be beneficial in counteracting drowsy driving ${ }^{7-10)}$ and in combating fatigue in operational settings ${ }^{11)}$. In addition, some studies have concluded that naps as short as $10 \mathrm{~min}$ significantly improve short-term alertness and performance ${ }^{12-14)}$. Most of the research to date, however, has focused on napping in either predictable shift work settings or in laboratory settings in combination with a sleep deprivation paradigm, thereby providing the researcher the ability to regulate the temporal

*To whom correspondence should be addressed. placement and duration of the nap. Few studies have examined napping behavior in an operational setting where irregular shifts are prevalent.

Unpredictable work schedules are a common occurrence in locomotive engineers. It has been shown that the widespread use of on-call scheduling with locomotive engineers results in work periods spread throughout the 24$\mathrm{h}$ day ${ }^{15,16)}$. Typically, most freight locomotive engineers in the United States are on-call $24 \mathrm{~h} /$ day and are usually called by their employer two hours prior to the time that they are required to report to work.

Irregular work schedules such as those experienced by many freight locomotive engineers can negatively affect sleep quantity and sleep quality, especially when the engineers are required to work at night. Previous studies have shown that the irregular work schedules used in the railroad industry can lead to decreased sleep lengths and decreased on-thejob alertness by the locomotive engineers ${ }^{17-19)}$. Due to the 
nature of on-call scheduling, freight engineers also experience days that are different from the $24-\mathrm{h}$ solar day ${ }^{19,20)}$. These days can either be less than $24 \mathrm{~h}$ or greater than $24 \mathrm{~h}$, depending upon the work and sleep activity of the engineer. "Short" days occur when the engineer phase advances his sleep, waking up earlier on one day than the day before (e.g., waking up at $7 \mathrm{AM}$ one day and then waking up at $5 \mathrm{AM}$ the next day). "Long" days occur when the engineer phase delays his sleep, waking up later on one day than the day before (e.g., waking up at 7 AM one day and then waking up at 9 AM the next day). These short and long days are experienced quite often and in heterogeneous fashion by locomotive engineers with about $25 \%$ of the days being less than $22 \mathrm{~h}$ long and about $28 \%$ of the days being longer than $28 \mathrm{~h}$ long ${ }^{19)}$.

Given the negative effect of irregular work schedules on locomotive engineers' sleep/wake habits, one might expect that they would be chronically partially sleep deprived and therefore would nap when the opportunity arose. In fact, daytime napping is reported in healthy adults even when they do not report being sleep deprived. One study concluded that as many as $75 \%$ of adults surveyed reported napping with nap durations ranging from 30 to $90 \mathrm{~min}$ long $^{21)}$. Another survey found that self-reported naps averaged about $73 \mathrm{~min}$ in length ${ }^{22}$. However, these surveys were one time events where the participants completed the survey by simply estimating his or her average nap length. In addition, these surveys did not take into account the potential effect of different types of work schedules on napping. To our knowledge, no study to date has examined the potential effect of napping on sleep in irregular work schedules.

The purpose of the current study was to examine napping behavior in locomotive engineers on irregular work schedules and to determine its impact on sleep. The current study addressed four specific questions concerning napping behavior in locomotive engineers:

1. How often and how long do locomotive engineers nap?

2. Are sleep length, sleep quality, and feelings of being well-rested different between days with naps and days without naps?

3. Is nap length related to either the length of the previous sleep episode or the subsequent sleep episode?

4. Is nap length related to sleep quality or feelings of being well-rested?

\section{Materials and Methods}

The current study is part of an on-going fatigue research effort supported by the Federal Railroad Administration's Human Factors Program in the Office of Research and
Development. The data were initially gathered and reported by the Volpe National Transportation Systems Center ${ }^{23}$. The study design was approved for human subjects and all participants completed a consent form at the time of the study administration.

\section{Participants}

Locomotive engineers from six major U.S. railroads volunteered to participate in the study between 1992 and 1994. A total of 179 engineers participated in the study (mean age \pm SD: $43.6 \pm 6.5 \mathrm{yr}$ ). The engineers worked under an assortment of irregular schedules; though most were on-call $24 \mathrm{~h}$ a day. When on-call, engineers were typically notified by a phone call from their employer two hours prior to the time when they were expected to report to work.

\section{Materials and procedures}

Participants provided information on their work and rest activity as part of a 14-day activity log. The log was designed such that each participant entered data for each day defined from midnight to midnight. The activity log was completed on work days and days off. As part of the logs, the participants provided information about the time that they got into bed with the intention of sleeping and the time they got out of bed after sleeping. The logs also provided the participants with a means to report subjective assessments of their sleep by assessing ability to go to sleep ( $1=$ easily to $5=$ not at all), ability to stay asleep ( $1=$ easily to $5=$ not at all), and wellrested feeling upon awakening ( $1=$ well rested to $4=$ not at all rested). All participants completed the "ability to stay asleep" and "well-rested upon awakening" measures. In addition, 124 of the 179 engineers completed the "ability to go to sleep" measure. Additional details on this particular instrument can be found in a prior report ${ }^{23)}$.

\section{Data analyses}

Before completing additional analyses, the data from the logs were reorganized from days defined as midnight to midnight to days defined by sleep episodes. The original design of the sleep log with days defined from midnight to midnight resulted in activities that took place at midnight being artificially split between two days. For example, if a participant fell asleep at $10 \mathrm{PM}$ and woke up at $6 \mathrm{AM}$, the sleep episode was divided between the two days. To control for this side effect of the original activity log design, each day was defined by the time that the engineer reported awakening from a main sleep episode. Main sleep episodes were defined as any sleep period at least four hours in length. In a few cases, decisions had to be made when there were 
either two or more similar length sleep episodes or when no sleep episode of at least four hours was reported for $36 \mathrm{~h}$ or more. In those cases, major sleep episodes were defined as the sleep episode that occurred at night. Sleep periods that were not classified as major sleep episodes were classified as naps. In most cases, this was a single sleep episode, but if multiple short sleep episodes were reported, they were summed up to represent total nap time.

The redefinition of the days in the data set caused the days to vary in length resulting in fewer sleep cycle days than were in the original data set. The original data set with days classified from midnight to midnight consisted of 2,506 d. The new, redefined data set had 2,020 sleep cycle days. The decrease in the number of days in the data set was due to two primary causes. First, some days in the data set were dropped as a result of missing data. For example, if a particular day did not contain complete sleep data resulting in an inability to determine sleep length, the data were not used. Second, the irregular work patterns of locomotive engineers resulted in numerous sleep cycle days longer than $24 \mathrm{~h}$.

The data were analyzed in SPSS. As the first step of the analysis, the data set was split into days where naps occurred and days where no naps occurred. Napping was reported in $17.3 \%$ of the days in the data set ( $350 \mathrm{~d}$ ) by 141 of the engineers. No napping was reported in the remaining 1,670 $\mathrm{d}$ in the data set. Independent $\mathrm{t}$-tests were conducted to compare the main sleep time and the subjective measures of ability to go asleep, ability to stay asleep and feeling wellrested upon awakening across the days where naps occurred and the days where no naps occurred. Pearson correlations were then completed on the sleep-related data to examine the relationships between naps and the main sleep episodes. Correlations were completed between reported nap length and the length of the main sleep episode before the nap, length of the main sleep episode after the nap, and the subjective sleep measures of ability to go to sleep, ability to stay asleep, and well-rested feeling upon awaking.

\section{Results}

On the days when napping occurred, the average main sleep episode was $6.8 \mathrm{~h}$ and the average nap was $2.9 \mathrm{~h}$ (Fig. 1). Thus, on napping days engineers reported an average of $9.8 \mathrm{~h}$ of total sleep time. On days when no napping occurred, engineers reported an average of $7.7 \mathrm{~h}$ of total sleep time during the main sleep episode (Fig. 1). The independent ttests indicated that engineers reported significantly more sleep time in the main sleep episode on no nap days than on

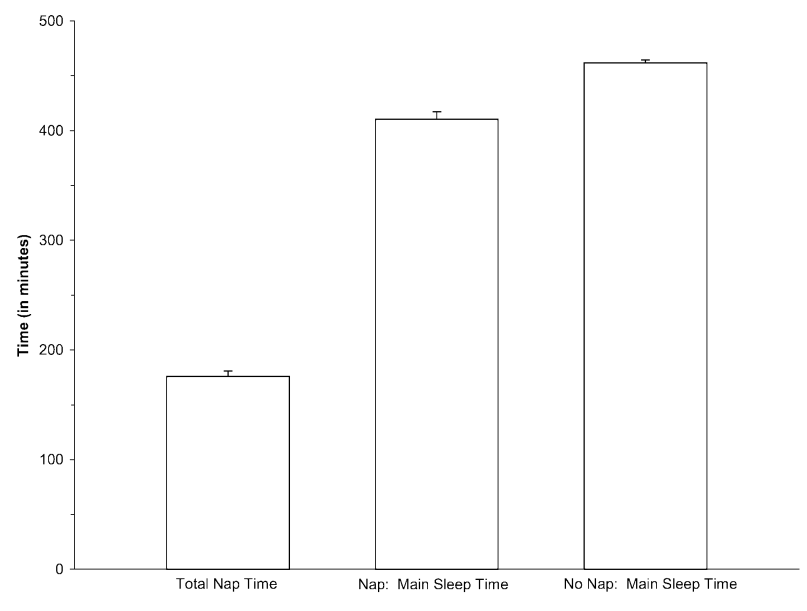

Fig. 1. Sleep Quantity.

Means (+ standard errors) of nap time and main sleep time on days with naps and main sleep time on days without naps.

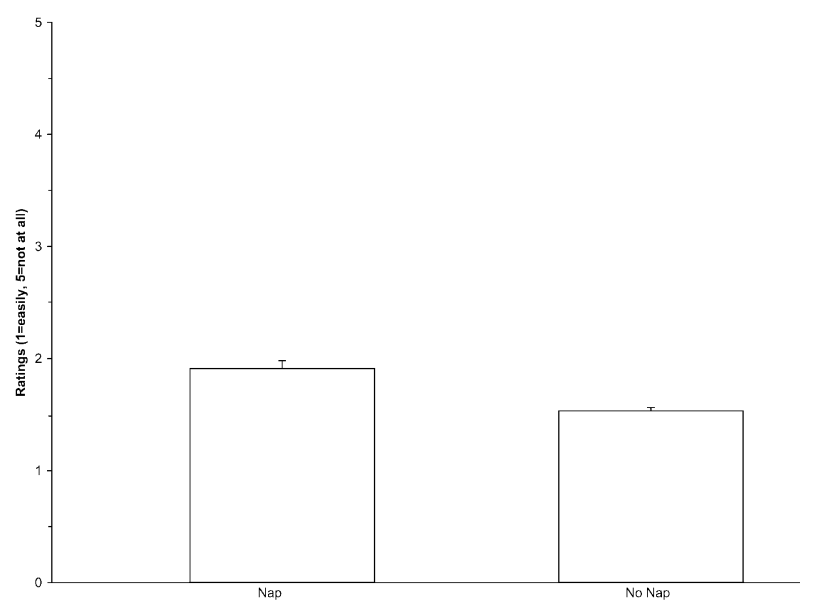

Fig. 2. Ability to Go to Sleep.

Means (+ standard errors) of ability to go to sleep for days with naps and days without naps.

nap days $(t=-6.73, \mathrm{df}=474.3, \mathrm{p}=.000)$, however, the engineers reported significantly more total sleep time on nap days (main sleep time plus nap time) than on no nap days $(t=13.45$, $\mathrm{df}=427.4, \mathrm{p}=.000$ ).

As shown in Fig. 2, the engineers reported being able to go to sleep somewhat more easily on no nap days than on nap days. The independent $t$-test indicated that the difference between the no nap days and nap days was significant $(t=4.84$, $\mathrm{df}=302.9, \mathrm{p}=.000$ ). In addition, the engineers reported being able to stay asleep more easily on no nap days than on nap days (Fig. 3). The t-test showed that there was a significant difference between the no nap days and nap days ( $t=5.55$, $\mathrm{df}=436.1, \mathrm{p}=.000$ ). Finally, as seen in Fig. 4 , the engineers 


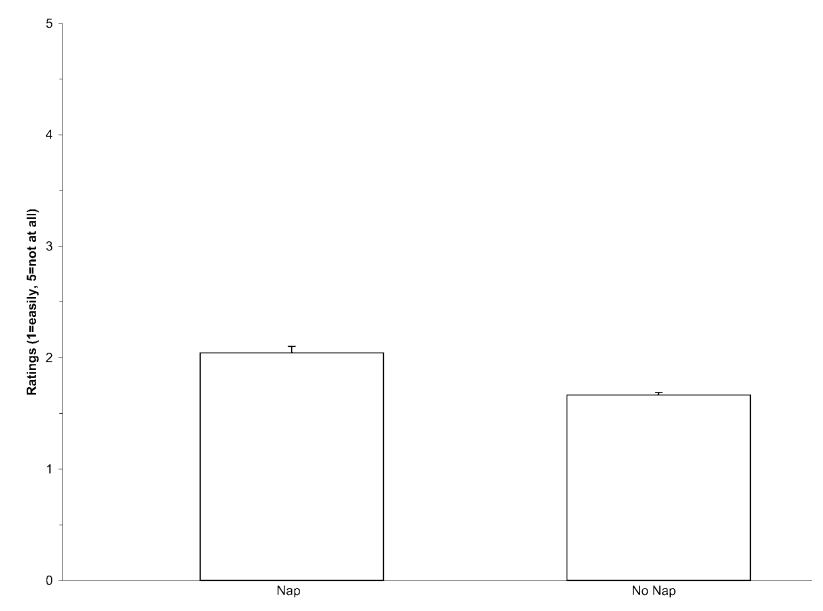

Fig. 3. Ability to Stay Asleep.

Means (+ standard errors) of ability to stay asleep for days with naps and days without naps.

reported a greater feeling of being well-rested upon awakening on no nap days than on nap days $(t=7.52$, $\mathrm{df}=451.0, \mathrm{p}=.000)$.

Although there were small but significant differences between sleep times and the subjective measures related to sleep between the no nap and nap days, the Pearson correlations showed that there was little relationship between nap time and the duration of the main sleep episode or between nap time and the subjective measures related to sleep (correlations ranged from $r=-.05$ to $r=.03$ ). These correlations included a comparison of the main sleep time before the nap to the nap duration as well as a comparison between the main sleep time after the nap to nap duration.

\section{Discussion}

The findings from the current study indicated that the locomotive engineers reported sleeping more on days when they chose to nap than on days when they did not nap, however their main sleep episode was shorter on the days that they napped. In addition, perhaps because of the increased time asleep across the day, the engineers reported a small but significant decrease in ability to go to sleep, ability to stay asleep, and a well-rested feeling upon awakening on days when they napped. It is interesting to note, however, that nap time was not significantly related to main sleep time prior to taking a nap, main sleep time after taking a nap, or the subjective sleep-related measures of ability to go to sleep, ability to stay asleep, and feeling well-rested upon awakening.

It is not surprising that when the engineers napped, they

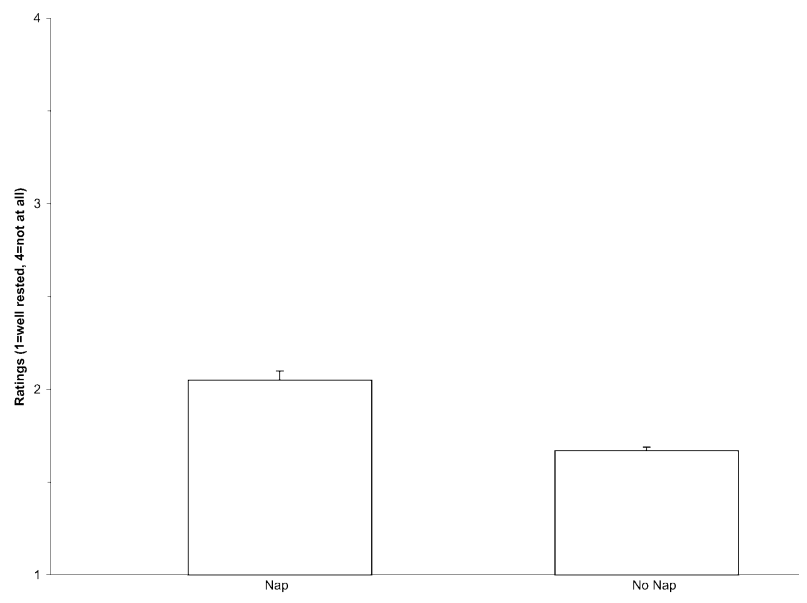

Fig. 4. Well-rested Feeling upon Awakening.

Means (+ standard errors) of well-rested feeling upon awakening for days with naps and days without naps.

reported more total sleep than when they did not nap. The reported total nap lengths by the engineers were quite long (almost $3 \mathrm{~h}$ long). One possible reason for long naps would be if the engineers were carrying a sleep debt from previous days. However, the length of the nap was not significantly correlated with the length of the main sleep episode prior to the nap. Given the nature of irregular work schedules, it is possible that the engineers were carrying a sleep debt over more than one day and that a decrease in sleep two to three days prior to the nap could have had an impact on the choice to take a nap and the length of the nap.

Because of the length of the naps, the slight decrease in ability to go to sleep, ability to stay asleep, and feeling wellrested upon awakening when compared to days where no nap occurred would be expected. It is important to point out that the decrease in these subjective variables, however, was quite small. Although it is impossible to tell from the current analyses whether these differences were functionally significant to the engineers, it seems unlikely. Therefore, it may have been worthwhile for the engineers to get extra sleep when they could, even if it meant that their sleep was not quite as efficient as it might have been if they would not have napped.

The lack of a relationship between nap time and the main sleep time subsequent to the nap indicates that even the long naps taken by the locomotive engineers did not result in a profound negative impact on their ability to sleep later that day. This would suggest that engineers may want to choose to nap to help recover some of the sleep that they may have lost due to their irregular schedule. It is also important to note that the subjective variables of ability to go to sleep, 
ability to stay asleep and well-rested feeling upon awakening were not related to nap length, supporting the idea that engineers may want to choose to nap when the opportunity arises. In fact, several U.S. railroad industries allow engineers to nap on duty under certain, restrictive conditions, such as idling on a siding while waiting for mainline traffic to pass.

It is interesting to compare the length of the naps reported by locomotive engineers to napping behavior reported by other adults. Locomotive engineers reported substantially longer naps than those generally reported by healthy adults in a one-time survey where adults reported napping for just over one hour a day ${ }^{22}$. Furthermore, a study asking healthy adults to record their sleep patterns over a 7-d period reported napping periods of only about $16 \mathrm{~min}^{24}$. This study examining napping across a 7-d period was quite similar in design to the current study, in that the adults were provided with an activity log and then sent home to go to work, take care of their family, and other social obligations. Thus, the difference between the napping periods reported by these two groups of adults may be attributed to the irregular work schedules of the locomotive engineers.

The conclusions in the current study may be somewhat limited due to the self-report nature of the data. In selfreport data, participants could purposely, unconsciously or accidentally complete the survey with incorrect information. In the current study, it is impossible to know if this happened. However, data were gathered from a relatively large, representative sample of engineers, including participants from six major railroad companies from around the United States to provide a robust data set. A related concern is that the participants were volunteers and may not have been representative of the true population of locomotive engineers. This concern was addressed in the same manner as the first, in that data were gathered from many engineers from different companies and from all over the United States. Finally, it is important to note that research in occupational settings is usually limited to self-reported data and to those individuals who volunteer to participate.

The current results suggest several future investigations. One potential study could examine whether nap length in irregular work schedules is related to sleep debt over several days. Another study of interest could examine whether the time that the nap took place had specific effects on the main sleep time. Other studies could investigate the potential effects of napping on work-related variables such as performance, attention to task, and on-the-job sleepiness.

In summary, the current results indicate that locomotive engineers slept more on days when they napped but had a shorter main sleep period following the nap. Napping also had a slight negative effect on sleep efficiency as measured by subjective reports of ability to go to sleep, ability to stay asleep and a well-rested feeling upon awakening. The negative effect of napping on sleep efficiency, however, was small. Thus, based on the current set of data, if engineers are willing to accept a slight decrease in sleep efficiency, they may want to choose to make an effort to nap more frequently.

\section{Acknowledgments}

The authors thank John K. Pollard for his efforts in the collection of the data used in the current study.

This research was supported in part by the Federal Railroad Administration's Office of Research and Development. The views of the authors do not purport to reflect the position of the Federal Railroad Administration or the U.S. Department of Transportation. This research was also based upon work supported by the National Science Foundation under Grant No. SES-0353698. Any opinions, findings, and conclusions or recommendations expressed in this material are those of the authors and do not necessarily reflect the views of the National Science Foundation.

\section{References}

1) Åkerstedt T, Torsvall L (1985) Napping in shift work. Sleep 8, 105-9.

2) Bonnet MH (1990) Dealing with shift work: physical fitness, temperature, and napping. Work Stress 4, 26174.

3) Rosa RR (1993) Napping at home and alertness on the job in rotating shift workers. Sleep 16, 727-35.

4) Bonnet MH, Arand DL (1994) The use of prophylactic naps and caffeine to maintain performance during a continuous operation. Ergonomics 37, 1009-20.

5) Dinges DF, Whitehouse WG, Orne EC, Orne MT (1988) The benefits of a nap during prolonged work and wakefulness. Work Stress 2, 139-53.

6) Gillberg M, Kecklund G, Axelsson J, Åkerstedt T (1996) The effects of a short daytime nap after restricted night sleep. Sleep 19, 570-5.

7) Horne JA, Reyner LA (1996) Counteracting driver sleepiness: effects of napping, caffeine, and placebo. Psychophysiology 33, 306-9.

8) Mitler MM, Miller JC, Lipsitz JJ, Walsh JK, Wylie CD (1997) The sleep of long-haul truck drivers. New Engl J Med 337, 755-61.

9) Reyner LA, Horne JA (1997) Suppression of sleepiness 
in drivers: combination of caffeine with a short nap. Psychophysiology 34, 721-5.

10) Macchi MM, Boulos $Z$, Ranney T, Simmons L, Campbell SS (2002) Effects of an afternoon nap on nighttime alertness and performance in long-haul drivers. Accid Anal Prev 34, 825-34.

11) Rosekind MR, Gander PH, Gregory KB, Smith RM, Miller DL, Oyung R, Webbon LL, Johnson JM (1996) Managing fatigue in operational settings I: physiological considerations and countermeasures. Behav Med 21, 157-65.

12) Takahashi M, Arito H (2000) Maintenance of alertness and performance by a brief nap after lunch under prior sleep deficit. Sleep 23, 813-9.

13) Tietzel AJ, Lack LC (2001) The short-term benefits of brief and long naps following nocturnal sleep restriction. Sleep 24, 293-300.

14) Tietzel AJ, Lack LC (2002) The recuperative value of brief and ultra-brief naps on alertness and cognitive performance. J Sleep Res 11, 213-8.

15) Foret J, Lantin G (1972) The sleep of train drivers: an example of the effects of irregular work schedule on sleep. In: Aspects of Human Efficiency. ed. by Colquhoun WP, 273-82, English Universities Press, Cambridge.

16) Pilcher JJ, Anderson J, Edwards G, Coplen MK (2002) Work- and sleep-related predictors of subjective onduty alertness in irregular work schedules. Transportation Research Record 1803, 16-21.

17) Kogi K, Ohta $T$ (1975) Incidence of near accidental drowsing in locomotive driving during a period of rotation. J Human Ergol 4, 65-76.

18) Torsvall L, Åkerstedt T, Gillberg M (1981) Age, sleep and irregular work hours. A field study with electroencephalographic recordings, catecholamine excretion and self-ratings. Scand J Work Environ Health 7, 196-203.

19) Pilcher JJ, Teichman HM, Popkin SM, Hildebrand KR, Coplen MK (in press) Effect of day length on sleep habits and subjective on-duty alertness in irregular work schedules. Transportation Research Record.

20) Pilcher JJ, Coplen MK (2000) Work/rest cycles in railroad operations: effects of shorter than 24-h shift work schedules and on-call schedules on sleep. Ergonomics 43, 573-88.

21) Dinges DF (1989) Napping patterns and effects on human adults. In: Sleep and Alertness: Chronobiological, Behavioral, and Medical Aspects of Napping. eds. by Dinges DF, Broughton RJ, 171-204, Raven Press, New York.

22) Dinges DF (1992) Adult napping and its effect on ability to function. In Evolution, Chronobiology, and Functions of Polyphasic and Ultrashort Sleep. ed. by Stampi C, 118-34, Birkhauser, Boston.

23) Pollard JK (1996) Locomotive Engineer's Activity Diary. Report Nos. DOT/FRA/RPP-9601, DOTVNTSC-FRA-96-12. Federal Railroad Administration, U. S. Department of Transportation.

24) Pilcher JJ, Michalowski KR, Carrigan RD (2001) The prevalence of daytime napping and its relationship to nighttime sleep. Behav Med 27, 71-6. 